\title{
Biotipo facial y la forma coronaria de los incisivos centrales superiores en estudiantes de Estomatología, Cusco, Perú
}

\section{Facial biotype and the coronary shape of the upper central incisors in stomatology students, Cusco, Peru}

\author{
Herbert Cosio, Luzcelly Aguirre, Liceth Lazo
}

\section{RESUMEN}

Objetivo: determinar la relación entre el biotipo facial y la forma coronaria de los incisivos centrales superiores en estudiantes de Estomatología de la ciudad del Cusco. Materiales y métodos: Estudio de tipo descriptivo y relacional. En una muestra de 144 estudiantes de 18 a 35 años de la Escuela Profesional de Estomatología de la Universidad Alas Peruanas Filial Cusco. Se hizo el registro fotográfico frontal en reposo, para establecer el biotipo facial y una fotografía intraoral, para la forma del Incisivo central superior. Se utilizó el programa Planmeca Romexis` para el análisis de las fotografías.

Resultados: con respecto al Biotipo facial el 65,28\% presento el biotipo Ovalado, el 25\% el cuadrado y el 9,72\% el triangular. La forma coronaria del incisivos central superior tuvo el 45,14\% la forma ovalada, el 28,47\% la cuadrada y el $26,39 \%$ triangular. No hubo asociación estadísticamente significativa $(\mathrm{p}=0,057)$ entre el biotipo facial y la forma coronaria del incisivo central superior. Conclusiones: el biotipo facial no está relacionado a la forma de los incisivos superiores en estudiantes de Estomatología de la ciudad del Cusco.

Palabras clave: estética facial, contorno facial, forma del incisivo (MeSH)

\section{ABSTRACT}

Objective: to determine the relationship between the facial biotype and the coronary shape of the upper central incisors in stomatology students from the city of Cusco. Materials and methods: Descriptive and relational study. In a sample of 144 students from 18 to 35 years of age at the Professional School of Stomatology of the Alas Peruanas Filial Cusco University. The frontal photographic record was made at rest, to establish the facial biotype and an intraoral photograph, for the shape of the upper central incisor. The Planmeca Romexis program was used to analyze the photographs.

Results: with respect to the facial biotype, $65.28 \%$ presented the Oval biotype, $25 \%$ the square and $9.72 \%$ the triangular. The coronary shape of the upper central incisors was $45.14 \%$ oval, $28.47 \%$ square, and $26.39 \%$ triangular. There was no statistically significant association $(\mathrm{p}=0.057)$ between the facial biotype and the coronary form of the upper central incisor. Conclusions: facial biotype is not related to the shape of the upper incisors in stomatology students from the city of Cusco.

Key words: facial aesthetics, facial contour, incisor shape (MeSH)

1. Cirujano Dentista UCSM, Magíster en Estomatología UPCH, Doctor en Educación UCSM, Docente a tiempo completo de la Universidad Alas Peruanas Filial Cusco

3. Cirujano Dentista UAC, Magister en Salud con mención en Salud Publica UANCV, Doctor es Odontología UAP, Docente a tiempo completo de la Universidad Alas Peruanas Filial Cusco 


\section{INTRODUCCIÓN}

El termino Biotipo facial permite la clasificación de los individuos en grupos, según ciertas variaciones de las proporciones del rostro en sentido transversal y vertical. Este conjunto de características morfodiferenciales son determinadas genética y ambientalmente. Son de uso básico para la ortodoncia, cirugía maxilofacial y la rehabilitación oral.

El biotipo facial se puede clasificar en dolicofacial con predominio del largo de la cara, braquifacial con predominio del ancho de la cara y mesofacial con equilibrio entre el acho y largo de la cara (Cerda et al., 2019). Fue Ricketts quien describió el concepto de biotipo facial, manifestando que es el conjunto de características morfológicas y funcionales (Sánchez \& Yañez, 2015).

El concepto de estética es subjetiva en relación a la belleza humana, se le puede dar muchas interpretaciones. Pero hay una parte objetiva de la estética, es el uso de las medidas, proporciones, simetría y armonía, que son usados para reproducir la belleza (Núñez \& Núñez, 2019).

En la estética de cara son importantes las proporciones faciales, las cuales se pueden obtener mediante la antropometría directa. La cual se basa en medidas lineales entre puntos anatómicos, que permiten obtener variables de tamaño y forma a partir de los puntos anatómicos previamente marcados (Onar et al., 2020). En la actualidad la antropometría se sigue usando para determinar las dimensiones de las estructuras anatómicas, y es mediante la fotografía y el análisis computacional es que se puede obtener (Suazo et al., 2008).

El contorno facial es una característica específica del rostro humano. Es analizado como parte del componente estético de los planes de tratamiento odontológico. En odontología legal y forense es usado como método de identificación. Se registran cuatro formas de contorno facial, el triangular, el cuadrado, el ovalado y el redondo. Esta variación del tipo de rostro es el resultado de la interacción de factores genéticos y ambientales (Acosta et al., 2011).

En la estética facial son los dientes anteriores son elementos importantes. Los incisivos establecen el atractivo en el rostro, los incisivos son dominantes en la sonrisa. Se sugiere que el contorno de estos debe de tener una proporción adecuada que se a agradable a a la vista y estén en equilibrio con la forma de la cara. Un factor determinante en la estética es la forma del diente que debe de ir acorde con la morfología facial (Weber et al., 2014). Es justamente el incisivo central superior el diente con mayor relevancia en la estética dental. Por lo tanto la elección de la forma es de vital importancia para la estética de la sonrisa y facial (Rodríguez et al., 2011).

Un parámetro importante para la estética facial es la sonrisa, donde los incisivos son elementos imprescindibles. Es así que Williams establece una propuesta para la elección de los Incisivos centrales de los maxilares (ICM) en los pacientes desdentados. Mediante la forma del rostro se puede establecer la forma del ICM, determinándola la "ley de la armonía" (Cabello \& Alvarado, 2015). La armonía dental y facial esta relacionada con las proporciones, que desde los egipcios se ha ido estudiando, posteriormente los griegos nos dejaron la proporción de oro o proporción divina. Esta proporción relaciona el equilibrio entre las diferentes partes del cuerpo (Ramírez et al., 2009).

Se ha categorizado la forma del ICM en la forma triangular, ovoide y cuadrada. Las cuales se podrían relacionar a la forma del rostro, la cual también puede tener las tres formas o contornos. Por otro lado, se manifiesta que en las poblaciones humanas existe una asociación entre la morfología dentaria con un patrón de variación geográfica (López et al., 2016).

El análisis de las fotografías digitales mediante el uso de software antropométrico es una herramienta en uso. Sirve para establecer el biotipo 
facial de manera digital, permitiéndonos hacer el análisis de las dimensiones y proporciones faciales en diferentes ámbitos de la odontología y la antropología física. En el mercado se pueden encontrar programas de uso comercial, de fácil manejo y de acceso libre (Suazo et al., 2008). El objetivo de la investigación fue determinar la relación del biotipo facial con la forma del contorno de la corona d ellos incisivos centrales superiores.

\section{MÉTODOS Y MATERIALES}

Estudio de tipo descriptivo, transversal y relacional, realizado en la Escuela Profesional de estomatología de la Universidad alas Peruanas Filial Cusco.

El muestreo fue probabilístico y la muestra estuvo constituida por 144 estudiantes de 18 a 35 años de edad, pertenecientes al grupo étnico mestizo. Para la recolección de los datos los participantes firmaron su consentimiento informado. Se mantuvo el anonimato de los participantes.

Para la selección de las unidades de análisis se tomó en cuenta los siguientes criterios: que no se encuentren o hayan recibido tratamiento de ortodoncia, que no tengan restauraciones estéticas, no presenten coronas y que no tengan anomalías craneofaciales que afecten la forma de la cara.

Para establecer la forma del rostro o biotipo facial se hizo el registro fotográfico frontal en reposo.

Para lo cual se le pidió al estudiante que asuma una posición natural de la cabeza y que los labios se encuentren reposos, no necesariamente en contacto. En esta posición los ojos deben de estar abiertos, el eje bipupilar paralelo al piso y el que el mentón no presentar hiperactividad.

Con esta primera toma se evaluó el tipo facial relacionando la altura y ancho de la cara, con lo cual se pudo establecer la forma triangular, cuadrada y ovalada.
Para establecer la forma de los dientes se hizo el registro fotográfico intraoral y mediante el análisis del contorno del incisivo central superior se pudo determinar la forma triangular, cuadrada y ovalada. En esta última fotografía se usó un abrebocas (Figura 1).

Se utilizó el programa Planmeca Romexis ${ }^{\oplus}$ para el análisis de las fotografías. Este software cuenta con un conjunto de herramientas para una mejor visualización de las imágenes.

Permite hacer mediciones, dibujos y anotaciones, por lo que nos permitió una mayor confiabilidad de las apreciaciones de la forma del rostro y de los dientes. Este programa permitió tener una proporción real de las medidas del rostro y dientes, el cual se hizo mediante un sistema de calibración.

Para la medición se usó los puntos cefalométricos a nivel de las cienes, el ancho bicigomático, correspondiente a la distancia entre los dos procesos cigomáticos y los puntos del gonion. $\mathrm{Al}$ unir estos puntos se observó la forma geométrica del rostro (Figura 2). Del mismo modo se evaluó el contorno de los incisivos, trazando líneas en las superficies proximales, pudiendo registrar las tres formas del diente.

\section{Figura $\mathrm{N}^{\circ} 1$ registro fotográfico con el abrebo- cas y uso de un calibrador milimetrado}

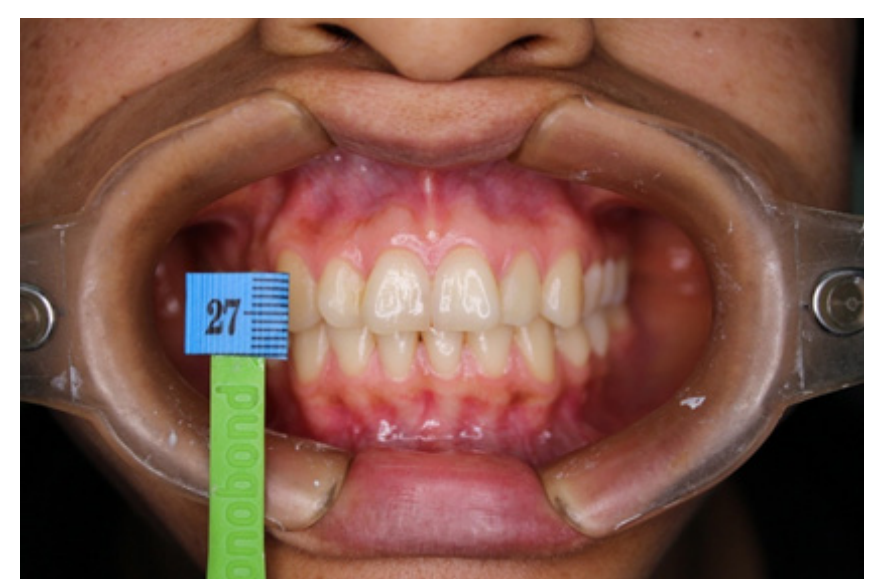


Figura $\mathrm{N}^{\circ} 2$ Uso del programa Planmeca Romexis

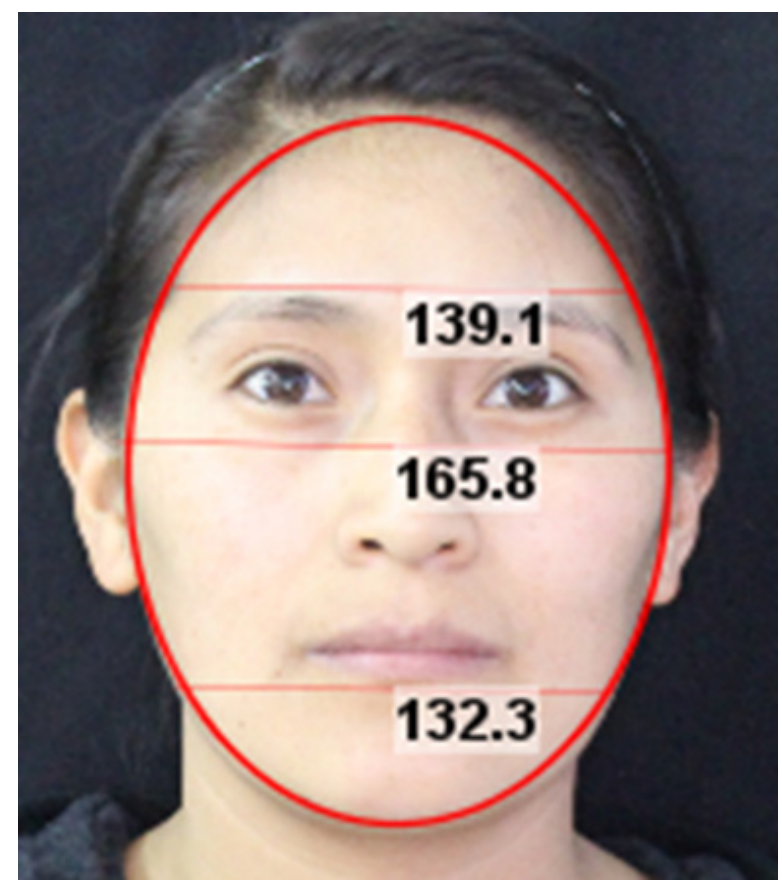

\section{RESULTADOS}

De una muestra de 144 estudiantes, el 42,4\% fue de sexo masculino y el $57,6 \%$ de sexo femenino. La edad media fue de 23,88 años, con un rango de edad de 18 a 35 años.

El biotipo facial fue ovalado en el 65,28\%, cuadrado en el $25 \%$ y triangular en el 9,72\% (Figura 3).

Con respecto a la forma coronaria del incisivo central superior fue ovalado en el $45,14 \%$, cuadrado en el $28,47 \%$ y triangular en el $26,39 \%$ ( $F i-$ gura 4).

Al relacionar el biotipo facial y la forma del incisivo central superior, se obtuvo un 33,3\% de estudiantes que presentaron el rostro ovalado y la forma de sus incisivos centrales ovalados.

El 11,1\% presento la forma del rostro cuadrado y la forma del incisivo central cuadrado.

El 2,1\% del total de estudiantes presento la forma del rostro triangular y la forma del incisivo central superior triangular (Tabla 1). Se aplicó la prueba estadística del Chi cuadrado, para ver la relación del biotipo facial y la forma del incisivo central superior, no encontrándose diferencias estadísticamente significativas con un valor $\mathrm{p}=0,057$.

\section{Figura $\mathrm{N}^{\circ} 3$ Biotipo facial de los estudiantes}

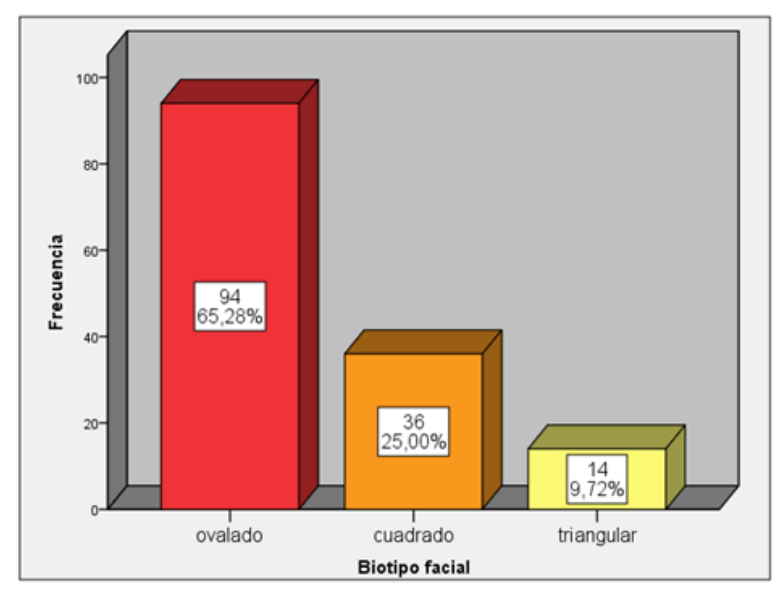

Figura $\mathrm{N}^{\circ} 4$ Forma coronaria del incisivo central superior de los estudiantes

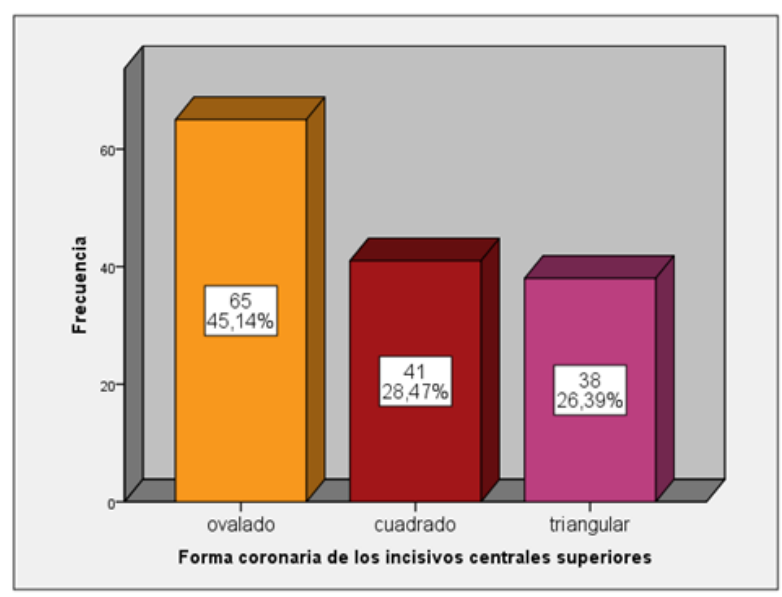


Tabla $N^{\circ} 1$ Relación del biotipo facial y la forma de la corona del incisivo central superior en los estudiantes

\begin{tabular}{|c|c|c|c|c|c|c|}
\hline & & & \multicolumn{3}{|c|}{$\begin{array}{l}\text { Forma coronaria de los incisivos centrales } \\
\text { superiores }\end{array}$} & \multirow[t]{2}{*}{ Total } \\
\hline & & & ovalado & cuadrado & Triangular & \\
\hline \multirow{6}{*}{$\begin{array}{l}\text { Biotipo } \\
\text { facial }\end{array}$} & \multirow[t]{2}{*}{ ovalado } & $\mathrm{N}$ & 48 & 19 & 27 & 94 \\
\hline & & $\%$ & $33,3 \%$ & $13,2 \%$ & $18,8 \%$ & $65,3 \%$ \\
\hline & \multirow{2}{*}{$\begin{array}{l}\text { cuadrad } \\
0\end{array}$} & $\mathrm{~N}$ & 12 & 16 & 8 & 36 \\
\hline & & $\%$ & $8,3 \%$ & $11,1 \%$ & $5,6 \%$ & $25,0 \%$ \\
\hline & \multirow{2}{*}{$\begin{array}{l}\text { triangula } \\
\mathrm{r}\end{array}$} & $\mathrm{N}$ & 5 & 6 & 3 & 14 \\
\hline & & $\%$ & $3,5 \%$ & $4,2 \%$ & $2,1 \%$ & $9,7 \%$ \\
\hline \multirow[t]{2}{*}{ Total } & & $\mathrm{N}$ & 65 & 41 & 38 & 144 \\
\hline & & $\%$ & $45,1 \%$ & $28,5 \%$ & $26,4 \%$ & $100,0 \%$ \\
\hline
\end{tabular}

${ }^{*} p=0,057$

\section{DISCUSIÓN}

Los incisivos superiores tienen características particulares que los convierten en piezas clave para individualizar a los pacientes. Las características como su forma pueden ser importantes para establecer registro e identificación a las personas.

Por otro lado, es una pieza clave en la estética facial, es importante registrar el tamaño en proporción al rostro, una simetría de formas entre el diente y el contorno facial, es decir una armonía con las líneas faciales. En nuestro estudio el biotipo facial no está asociada a la forma del contorno de la corona de los incisivos centrales superiores en estudiantes de Estomatología de la ciudad del Cusco.

Este resultado es consistente con otros estudios realizados por Weber et al. (Weber et al., 2014), Cabello y Alvarado (Cabello \& Alvarado, 2015) y Acosta et al. (2011), quienes manifestaron que no hubo relación entre las medidas dentaria y faciales. Sin embargo, de manera contraria a lo establecido en la "Ley de la armonía" de Williams, que establece la armonía de las líneas paralelas de los dientes y el contorno facial. A un rostro cuadrado le correspondería incisivos con super- ficies proximales paralelas. A un rostro ovalado le corresponde incisivos con superficies proximales curvas o convexas. $\mathrm{Y}$ aun rostro alargado le correspondería incisivos con superficies proximales convergentes.

El contorno facial es producto de la interacción de factores genéticos y ambientales. En nuestro estudio el biotipo facial más frecuente es el ovalado, seguido del cuadrado y el menos frecuente es el triangular. Nuestros resultados son parecidos a lo descrito por Acosta (2011).

Cada grupo dentario realiza una función diferente. Los incisivos son determinantes en la estética del rostro, además de preservar el equilibrio y la armonía del arco dentario. En la forma de los dientes los limites externos curvos y redondeados determinan la forma ovoidea, la disposición divergente del límite externo del contorno determina la forma triangular y la disposición paralela del límite externo determina la forma cuadra.

La valoración de estas formas se realizó en forma objetiva mediante el uso de imágenes analizadas en programas computarizados. En nuestro estudio la forma coronaria del incisivo central superior más frecuente es la ovalada. Resultado similar a lo descrito por Weber et al. (2014) y Cabello y Alvarado ( 2015). 
En la odontología forense los dientes sirven para fines de identificación, gracias a que poseen características especiales que permiten su registro morfológico. Estas son formas fenotípicas producto del proceso indirecto de secreción mineral durante la odontogenesis.

La forma del rostro es una característica que el profesional de la Odontología podría utilizar como parámetro referencial al momento de elegir la forma de los dientes incisivos en las prótesis fijas o removibles. Pero sería mejor que al momento de elegir la forma del diente anterior, tener en cuenta la experiencia y el sentido critico del profesional.

También se debe de tener en cuenta el deseo del paciente. Todo esto con el fin de alcanzar un resultado estético y aceptado por el paciente. La muestra podría ampliarse en futuras estudios y establecer la relación de la forma de la cara con la forma del arco dentario en una población con mas criterios de selección.

\section{CONCLUSIONES}

En los estudiantes de la Escuela Profesional de Estomatología de la Universidad Alas Peruanas Filial Cusco, predomino el biotipo facial ovalado y en la forma coronaria de los incisivos superiores predomino la ovalada.

Al hacer el cruce de variables no se encontró asociación estadísticamente significativa, por lo que concluimos que no está relacionado biotipo facial con la forma coronaria de los incisivos centrales superiores.

\section{REFERENCIAS BIBLIOGRÁFICAS}

Acosta, D., Porras, A. M., \& Moreno, F. (2011). Relation between the facial contour form, the dental arches and the upper central incisors shape in dental students from Universidad del Valle-Cali. Revista Estomatología, 19(1), 8-13.

Cabello, M., \& Alvarado, S. (2015). Relationship between the shape of the upper central incisors and the facial contour in dental students. Lima. Peru. Journal Of Oral Research, 4(3), 189-196. https://doi.org/10.17126/joralres. 2015.038

Cerda, B., Schulz, R., López, J., \& Romo, F. (2019). Parámetros cefalométricos para determinar biotipo facial en adultos chilenos. Revista clínica de periodoncia, implantología y rehabilitación oral, 12(1), 8-11.

López, S., Soto, C., Aramburú, G., Rodríguez, I., Cantín, M., \& Fonseca, G. M. (2016). Investigación de Rasgos Dentales No Métricos en Poblaciones Sudamericanas Actuales: Estado de Situación y Contextualización Forense. International Journal of Morphology, 34(2), 580-592.

Núñez, G., \& Núñez, J. G. (2019). Perfilografía áurea: Estética de las proporciones nasales y faciales. Cirugía Plástica Ibero-Latinoamericana, 45(2), 127-138.

Onar, V., Siddiq, A. B., Asal, R., \& Parés, P. M. (2020). Los Tipos Craneométricos Caninos Aparecen Bien Expresados a Nivel de Conformación del Arco Cigomático. International Journal of Morphology, 38(1), 78-82.

Ramírez, R. A., Setién, V. J., Orellana, N. G., \& García, C. (2009). Microfiltración en cavidades clase II restauradas con resinas compuestas de baja contracción. Acta Odontológica Venezolana, 47(1), 131-139.

Rodríguez, P., Martínez, J. A., Celemín, A., Romeo, M., \& Rivero, A. (2011). Estudio entre las formas y proporciones del contorno facial y del incisivo central. Gaceta Dental, 228, 90-105.

Sánchez, M. A., \& Yañez, E. E. (2015). Asociación entre el biotipo facial y la sobremordida: Estudio piloto. Revista Estomatológica Herediana, 25(1), 05-11.

Suazo, I., López, X., \& Márquez, V. (2008). Software Antropmeter, una Nueva Herramienta para Análisis Facial. International Journal of Morphology, 26(4), 973-974.

Weber, B., Fuentes, R., García, N., \& Cantín, M. (2014). Relaciones de Forma y Proporción del Incisivo Central Maxilar con Medidas Faciales, Línea Mediana Dentaria y Facial en Adultos. International Journal of Morphology, 32(3), 1101-1107. 